\title{
Early Warning and Response System (EWARS) for chikungunya, zika and dengue outbreaks: The innovated WHO-TDR tool.
}

Rocio Cardenas ( $\square$ rocio.cardenas@students.uni-freiburg.de)

Albert-Ludwigs-Universität Freiburg Medizinische Fakultät https://orcid.org/0000-0001-8916-8544

Laith Hussain-Alkhateeb

Goteborgs universitet Sahlgrenska Akademin

David Benitez-Valladares

Ciudad de Mexico Secretaria de Salud

Gustavo Sanchez Tejeda

Secretaria de Salud de Mexico

Axel Kroeger

Special Programme for Research and Training in Tropical Diseases

Research article

Keywords: zika, chikungunya, dengue outbreak, early warning, Colombia, Mexico

Posted Date: December 10th, 2019

DOI: https://doi.org/10.21203/rs.2.18483/v1

License: (c) (7) This work is licensed under a Creative Commons Attribution 4.0 International License. Read Full License 


\section{Abstract}

Background. In the Americas, endemic cities for Aedes-borne diseases such as chikungunya, Zika and dengue face great challenges particularly since the recent outbreaks of CHIKV and ZIKV, all transmitted by the same insect vector Aedes aegypti and albopictus. Areas, such as Colombia and Mexico with the highest incidence and most frequent outbreaks of the three diseases are located in tropical environments due to their favorable eco-epidemiological conditions for vector breeding. In Colombia, the city of Cúcuta on the border with Venezuela is one of such highly endemic areas. Likewise, in Mexico a number of municipalities has very similar environmental conditions. This is why these urban areas provide the opportunity to test the Early Warning and Response System (EWARS), developed originally for dengue outbreaks, also for the other two diseases (Chikungunya and Zika).

Methodology. Through the retrospective analysis of epidemiological, climate and entomological data produced by the national surveillance systems in Colombia and Mexico, we intended to predict outbreaks with a high sensitivity and positive predictive value (PPV) through alarm signals by using the EWARS tool. The registered outbreaks of DENV 2012-2016, CHIKV 2014-2016 and ZIKV 2015-2016 were analyzed for 2 years retrospectively ("run in period") and one year of analysis ("evaluation period"). Outbreak prediction for dengue and Zika was for both countries but for Chikungunya in Colombia only due to the availability of surveillance data.

Results. In Mexico, the sensitivity of different alarm signals for correctly predicting an outbreak ranged between 74-92\% for dengue, 77-93\% for chikungunya and $78-97 \%$ for Zika. Their Positive Predictive Values ranged between $51-68 \%$ for dengue, $48-92 \%$ for chikungunya and $11-100 \%$ for Zika. The lag time between predictions and start of the outbreak (i.e. the time available for early response activities) was for dengue 3-5 weeks, for chikungunya $10-13$ weeks and for Zika 3-5 weeks.

Conclusion. The implementation of an early warning and response system (EWARS) could substantially reduce the magnitude and occurrence of outbreaks and the elevated social and economic toll.

\section{Introduction}

Dengue, Zika and Chikungunya arboviruses spread to humans through the bite of an infected Aedes aegypti or Ae. albopictus mosquito and present a growing public health threat to endemic countries. The arbovirus diseases produced by the dengue virus -DENV, chikungunya virus -CHIKV and zika virus -ZIKV, present risks of transmission in 3.9 billion people living in 128 countries of the tropical and subtropical areas of the world $(1,2,3,4)$. Currently there is no specific pharmacological treatment nor an effective vaccine for public health use. Although these diseases present a high proportion of subclinical cases, they are characterized by high morbidity and have common symptoms that can affect the clinical diagnosis (5).

These diseases have a high social and economic impact, particularly when they appear as epidemics. An estimate of the economic costs of a chikungunya outbreak in Colombia 2013 showed an approximate cost of 100 million dollars, equivalent to $4.0 \%$ of the national gross domestic product (GDP) (6); estimates for dengue outbreak costs in 2011 were 12 million US\$ in Vietnam, 6.75 million US\$ in Indonesia, 4.5 million US\$ in Peru and 2.8 million US\$ in Dominican Republic (all in 2012 US\$) (7). It was recently estimated that between 2015 and 2017, Zika virus outbreaks caused losses of 7 to 18 Billion dollars, being the direct and indirect costs associated with microcephaly and Guillain-Barré syndrome the greatest long-term cost (8).

Countries endemic for Aedes-borne viral diseases and countries with low-level or no transmission are threatened by outbreaks which are detected late and where the response mechanisms are often inadequate. Early detection of outbreaks poses a challenge, since no universally accepted or proven sets of early warning indicators exist. Although the transmission of Aedes-borne viral diseases could be substantially reduced by effective vector control interventions, late detection and inadequate response mechanisms are compounding the effects of rapid transmission. A systematic review showed that practically no country uses outbreak forecast (prediction) $(9,10)$. In contrast, a number of countries uses early outbreak detection tools based on the increase of case numbers above a pre-established threshold (meaning that the outbreak has started already). With this in view, the Special Program for WHO/TDR initiated together with European research institutions and national dengue control services and academia in endemic ten partner countries, the development of a web-based Early Warning and Response System (EWARS) for dengue, with potential uses for other arbovirus disease outbreaks. An initial test was performed in a retrospective study in five endemic countries (Brazil, Mexico, Dominican Republic, Vietnam, Malaysia) $(11,12,13)$. Subsequently, based on user recommendations and inputs from expert panels, an innovative web-based EWARS tool (dashboard) was developed and tested in three endemic countries (Brazil, Mexico and Malaysia) to evaluate its qualitative and quantitative performance and user friendliness (14).

However, EWARS has never been tested for other emerging Aedes-borne diseases, such as Zika and chikungunya and their epidemiological findings against dengue outputs are yet lacking which hinders important policy making and early response activities during disease outbreaks. This study therefore aimed at presenting and discussing some epidemiological implications of dengue, chikungunya and zika outbreaks in highly-endemic settings while demonstrating the use and performance of EWARS for wider arbovirus disease outbreaks.

\section{Method}

\section{Description of study sites}

In Colombia, Cucuta, the capital of North Santander state ("department") was selected which has around 750000 inhabitants, $1176 \mathrm{Km}^{2}$ in area size and located $320 \mathrm{~m}$ above sea level at latitude $07^{\circ} 53^{\prime} 00^{\prime \prime} \mathrm{N}$, longitude $72^{\circ} 30^{\prime} 19^{\prime \prime} \mathrm{W}$. The climate is warm and characterized by temperatures ranging between 21 and $36^{\circ} \mathrm{C}$; an average annual rainfall of $655 \mathrm{~mm}$ and an annual average relative humidity between 70 and $75 \%$ (15). While dengue outbreaks have a longer history in Cúcuta, outbreaks of CHIKV and ZIKV were reported after 2014 and 2015, respectively. 
In Mexico, 137 highly endemic urban municipalities in tropical areas in 28 states of the country with a high incidence of the three arbovirus diseases have been selected.

\section{Public Health Surveillance system}

In Colombia, the National Public Health Surveillance System (SIVIGILA) provides systematic and timely information on the dynamics of dengue, Zika and Chikungunya. The data reporting units belong to the public and private health system. The data was obtained from SIVIGILA at the IDS (Departmental Health Institute), with certified authorization

In Mexico, the national surveillance system includes permanent notification of clinical cases of dengue, Chikungunya and Zika, birth defects into the online platform SINAVE (National Epidemiological Surveillance System) as well as entomological data (ovitrap indexes) and response activities (into the online entomological platform). Both platforms are fed on a weekly basis with the information from all health centers (public and private).

\section{Data collection of outbreak and alarm indicators}

In Cucuta/Colombia, the study has covered the period from January 1, 2012 to December 31, 2017 including all reported cases of dengue where included using the standardized case-definitions by the Ministry of Health $(\mathrm{MoH})$ and the INS. This study analyses part of the total number of cases of the Chikungunya and Zika outbreak in the urban area of Cúcuta, only those corresponding to the individualized notification of SIVIGILA (16, 17). In Mexico the data collection started also in January 2012, however, the analysis period lasted until December 2018. The data set has been provided by SINAVE and by the entomological online platform from the Secretary of Health.

For the application of EWARS, the temporal unit was defined as the epidemiological 'week' (from Sunday to Saturday) and the spatial unit was based on preexisting administrative units (municipalities or districts). At least three years of surveillance data records were retained for the EWARS analysis including a variable indicating the 'population size' of the corresponding district/localities.

Surveillance data on dengue, Zika and chikungunya were recorded as well as the individuals' place of residence, age, sex, date of onset of symptoms, date of case registration at the health center or hospital and the type of case ('probable', 'confirmed' by laboratory or by clinical symptoms and 'hospitalized' cases).

Weekly information of potential key alarm indicators was collected from each study district/municipality and included a candidate list of epidemiological, entomological and meteorological variables:

- Meteorological: mean outdoor air temperature, rainfall and relative humidity

- Epidemiological: patients' age, and probable cases)

- Entomological: positive ovitrap, average egg count per trap

\section{Data analysis}

Analysis by the EWARS tool:

The general methodological and operational principles of how EWARS processes the calibration and evaluation analysis including the generation of best-fitted prediction algorithm have been illustrated in details elsewhere $(9,12,14)$. In general, and during the retrospective phase of EWARS, the average number of cases of an arbovirus disease - within the expected "normal" or seasonal range illustrated in the endemic channel - are calculated for a fixed time period and with the use of a given z-value (which is the multiplier of the standard deviation of the moving average of weekly case numbers) during the calibration session, which generate a smoothed Endemic Channel (moving average $+(Z * S D)$ ). Weekly cases exceeding this Endemic Channel are said to be an outbreak. In this retrospective phase, the algorithm and all parametric coefficients needed for calculating the outbreak probability are computed: these coefficients depend primarily on the sensitivity (i.e. the proportion of correctly predicting an outbreak out of all outbreaks) and positive predictive value, PPV (i.e. the proportion of correct alarms out of all alarms) as direct measures for deciding the best calibrated settings (i.e. those with highest sensitivity and PPV). In the prospective stage of EWARS, an alarm signal (early prediction) is triggered when the 'outbreak probability' crosses the proposed 'alarm threshold' once prospective weekly information on the relevant alarm indicator(s) are fed into the system. Accordingly, instant numerical and graphical demonstration and interpretation of possible outbreak and its corresponding response plan is illustrated to the user at a given lag week (time between the prediction and an outbreak to occur).

Based on recommendation from previous reports (14), 'hospitalized' cases showed to be best outbreak indicator for the prediction of a forthcoming outbreak using EWARS. However, where the proportion of "mild" cases is high and patients are rarely hospitalized (such as in chikungunya and zika), 'probable' or 'confirmed' cases were used as outbreak indicators. In this study, z-values and alarm thresholds were determined for chikungunya and dengue via both manual and automatized procedures (which can assess the calibration settings via a 1000 iteration process per district). For zika data only the automatized version was employed. On the other hand, due to inadequate dengue records and inconsistent outbreak trend observed in the Colombian data, a data normalization step was performed prior to the application of the EWARS tool.

In Mexico districts with at least three years' records of at least $\geq 5$ cases per evaluation period were included ( $N=137)$. Only districts with no meaningful parameters and outputs from the final results such extreme z-outbreak, thresholds and window sizes or where no convergence was reached or poor calibration match were dropped. Descriptive presentations of the sensitivity, PPV and lag weeks as well as outbreak parameters derived from the evaluation stage were calculated and presented. In the case of Mexico, average values obtained from multiple districts were presents in the final results after rounding off to 1.0. 
Descriptive statistics, of both graphics and estimates presentations, was sought in this paper. To give an overview of the pattern of chikungunya and Zika occurrence in Colombia, disease incidences were determined with the cases from the urban area included for this analysis ( $\mathrm{n}$ cases / 100,000 population).

\section{Ethical aspects:}

This study analyzed only secondary data obtained from Colombian and Mexican institutions with the authorization of the Surveillance System (Official Communication 23.02.2018). Ethical endorsement was obtained from the Ethics Committee of the University of Freiburg ( $\left.\mathrm{N}^{\circ}-145 / 18\right)$ which was approved by local health authorities.

\section{Results}

\section{Epidemiological features of outbreaks}

Data sets were analysed to describe outbreaks of dengue, chikungunya and Zika in municipalities in Colombia and Mexico during the period 2012-2018. In general, dengue records were analysed for the five-year period in Colombia and seven years in Mexico. However, chikungunya data taken into account for this study were three years in Colombia (2014-2016) and five years in Mexico (2014-2018). The Zika data included in this analysis correspond to three years in Colombia (2015-2017) and four years in Mexico (2015-2018).

\section{Dengue}

In Colombia, for the urban area of Cucuta 2013 was an epidemic year for dengue, with 5049 cases ( 819.94 cases per 100,000 population), followed by the 2014 outbreak with 4900 cases (787.82 cases per 100,000 inhabitants). During the period 2014-2016 a decrease in cases was observed, with a slight increase after the start of the zika outbreak. In Mexico, 2012 and 2013 were epidemic years for dengue with 53029 and 64996 confirmed cases respectively. After those epidemic year Mexico has seen a continuous reduction of dengue cases until 2018 with 12706 confirmed cases.

\section{Chikungunya}

Regarding chikungunya during the weeks 44 of 2014 and 5 of 2015 the surveillance system in Colombia recorded the largest number of cases, with attack rates of 114.96 cases per 100,000 population in 2014 and 20.38 cases per 100,000 population in 2015 . The highest peak was observed near the 49 th week of 2015. The outbreak ended after week 9 in 2015 but there were still 128 cases in 2016. In Mexico, although the first cases appeared during 2014 (222 in the whole country) the chikungunya outbreak occurred during 2015 with 12,588 confirmed cases. After 2015 there has been a steady reduction with 759 confirmed cases in 2016 until only 39 confirmed cases in 2018. The peak of the 2015 chikungunya outbreak occurred around week 29.

\section{Zika}

The Zika outbreak in the municipality of Colombia presented an increase from week 51 of 2015 until week 10 in 2016 (12 weeks). The peak of cases occurred in week 1 of 2016 (753 cases), and a total of 4576 cases during the years 2015 and 2016. The highest attack rate was 691.14 cases per 100,000 population. In Mexico the Zika outbreak, after some sporadic cases in 2015, occurred during 2016 with 8,588 confirmed cases. 2017 also presented a Zika outbreak but considerably smaller, 3,260 confirmed cases. During 2018 the trend continued and there were only 881 confirmed cases.

Variations of incidence rates occurred when dengue was the only arbovirus but after the arrival of CHIKV and ZIKV this changed. In the 2013 dengue outbreak, the highest attack rates in the city were 819.94 cases per 100,000 population. When two of those arboviruses occurred simultaneously, dengue rates decreased to 215.19 cases per 100,000 population. With the dataset from individualized notification only, chikungunya rates were the lowest, while Zika reached a rate of 691.14 cases per 100,000 population.

\section{Findings from the EWARS application}

The datasets of all three arbovirus disease outbreaks were processed using the EWARS tool. The summary of the model calibrations, parameters including the sensitivity, PPV and lag weeks for each disease per country are presented in tables $1-3$. Number of 'outbreaks' and 'alarms', from which the sensitivity and PPV values were derived, are displayed in tables of results. Furthermore, the alarm thresholds were also illustrated to inform of expected ranges to use during the calibration process, independently for each disease and per each alarm indicator.

\section{Dengue}

A value of $z=1.0$ was found to be best to define the upper limit of the endemic channel using the hospitalized dengue cases as the outbreak indicator. The sensitivity to correctly predict a dengue outbreak varied between $74-92 \%$ whereas the PPV ranged between $50-68 \%$, including both single and multiple alarm prediction analysis whereby the multiple alarm prediction enhanced the prediction model. The lag time ranged from 3 to 5 weeks ahead of an outbreak. Table 1 illustrates more detailed results.

\section{Chikungunya}

Unlike the Colombian dengue data, the chikungunya data did not require any normalization treatment prior to the EWARS analysis. Due to insufficient hospitalized cases found in the Colombian surveillance dataset, the 'probable cases' were used as the outbreak indicator instead, which revealed a sensitivity range of $77-93 \%$ and PPV fluctuation of $48-92 \%$. The lag time between positive alarm and start of the outbreak was 10 to 13 weeks, much higher than that observed with dengue (Table 1).

\section{Zika}


Estimates of both sensitivity and PPV were derived which ranged between $50 \%-100 \%$ and $11 \%-100 \%$ in Colombia and between $78 \%-97 \%$ and $77 \%-100 \%$ in Mexico, respectively (Table 3). In general, the alarm indicators in Mexico outperformed those in Colombia of which both 'rainfall' and 'humidity' indicators ranked top in the list to predict an outbreak, whereas average egg factor was the lowest in the rank. Although the alarm and outbreak window sizes were comparable between both countries and across all alarm indicators, higher number of defined outbreaks and alarm thresholds were observed in Mexico in relation to Colombia. In contrast, broader lag times were shown in the Colombian data which ranged from 6 to 10 weeks compared to 4 to 5 weeks in Mexico.

\section{Discussion}

\section{Outbreak patterns}

As contextual information for the need of early outbreak warning, the outbreak pattern of the three diseases in Colombia and Mexico is shown in Figure.1. It illustrates that outbreaks of dengue, chikungunya and Zika are not independent in time, although they present differences in transmission peaks. During the first weeks of the chikungunya outbreak in Colombia, there was an unusual increase of dengue cases with 172 cases in week 44 of 2014 . After week 45 chikungunya exhibited the highest transmission peak and dengue cases descended rapidly. This can be explained by possible mis-diagnoses, taking into account that during the first phase of the chikungunya outbreak the presence of the virus in the city was not confirmed. In Colombia the samples of suspected patients were sent at the central level. Approximately two weeks later, confirmation of the presence of CHIKV in Cucuta and dissemination to all case reporting units was achieved. After that the increase of the correct chikungunya diagnosis started.

\section{Figure 1. Time series of dengue, chikungunya and Zika in the urban area of Cúcuta, Colombia 2012-2016}

Figure 1. Time series of analysed cases of Dengue, Chikungunya and Zika in the urban area of Cúcuta, Colombia during the period 2012-2016. Dengue is in blue bars; yellow line is Chikungunya and red line is Zika. The cases of dengue are quantified on the right. $\left(^{*}\right)$ Week of the first case of Chikungunya; $(+)$ Week of the first case of Zika).

The same happened when the Zika outbreak occurred. Two weeks before the highest peak of Zika cases, there was an increase in "dengue cases". Many of these may have been Zika. It seems that after the laboratory confirmation of the Zika virus in the city, the correct diagnoses and the number of cases increased.

A similar but less pronounced phenomenon was observed in Mexico in 2014/15: At the beginning of the chikungunya outbreak there was an increased dengue activity probably because the laboratory confirmation of CHIKV was not yet well established. The same happened with the Zika outbreak in 2016 when first dengue case numbers increased and subsequently Zika was detected (Figure 2). The delay in the confirmation of cases can lead to the silent spread of the disease in the different areas which makes control difficult. Therefore, the need for an improved surveillance system and for early outbreaks warning.

\section{Figure 2. Time series 2009-2019 of cases reported weekly in Mexico: dengue, chikungunya and Zika}

Figure 2. Time series of cases reported weekly in Mexico, of cases of dengue, chikungunya and Zika during the period 2009-2019. Dengue is in blue bars; yellow line is chikungunya and red line is Zika. The cases of Zika are quantified on the right. (*) Week of the first case of chikungunya; $(+)$ Week of the first case of Zika)

\section{Early Warning and Response System (EWARS)}

With the purpose of contributing to mitigate the effects of arbovirus outbreaks the EWARS applied in our study proved to be an adequate tool to predict dengue outbreaks 3 to 5 weeks, chikungunya outbreaks 10 to 13 and Zika outbreaks with 4 to 10 weeks ahead of the outbreak. This will allow the public health service to respond more efficiently through the timely allocation of resources and particularly vector control.

In the case of dengue, the sensitivity of alarm indicators to correctly predict an outbreak varied in both countries from 74 to $92 \%$, being the combination of meteorological indicators the alarm signal with the highest sensitivity. This is similar to that of other countries that are using EWARS for outbreak detection (83-99\% in Brazil, 50-99\% in Malaysia and 79-100\% in Mexico) (14). Likewise, the PPV of 50-68\% was similar to those reported in the other countries (40-88\% in Brazil, $71-80 \%$ in Malaysia and 50-83\% in Mexico) (14). Therefore, the EWARS would detect, about 9 out of 10 outbreaks and 7 out of 10 alarms will be correct alarms.

In the case of chikungunya, the sensitivity to correctly predict an outbreak varied among alarm indicator from 77 to $93 \%$, being the rain fall and the combination of meteorological indicator the alarm signals with the highest sensitivity. Likewise, the PPV of 48-85\% was similar to those reported with dengue in other countries (14). Therefore, the EWARS would detect outbreaks 10 to 13 weeks in advance, providing adequate time to activate response actions.

In the case of Zika, the sensitivity (50-100\%) and PPV (11-100\%) was similar to the prediction of dengue and chikungunya when using alarm indicators with the highest sensitivity and PPV-values.

It is the first time that EWARS has been evaluated with chikungunya datasets, and the results were optimal to recommend the implementation. Although only data for three years were analyzed, these results show that EWARS is applicable to Aedes borne arbovirus diseases. As the user-friendliness of the application has already been established (14), the next step is to bring it to practical use in endemic countries and monitor its ability to predict outbreaks and trigger effective response.

\section{Limitations of the study}


This study was based on the cases registered by the public health surveillance system in Colombia, which classifies the cases as probable, confirmed and hospitalized (17). However, some differences are detected with the definitions suggested by the WHO in relation to confirmed cases. All confirmed cases should present a positive laboratory test, but in the current database the confirmed cases were sometimes based on clinical criteria only. This reduces the reliability of the case definition.

For these reasons, in some periods there was a greater number of confirmed cases than of hospitalized cases, showing that the confirmed cases correspond often to outpatients. For dengue, the use of hospitalized cases as outbreak indicator was feasible, but due to the low proportion of "hospitalized cases" in chikungunya and Zika this was hardly the case; therefore, some authors suggest the use of probable and confirmed cases outbreak indicators (14, 18). The limitations described above were overcome by taking into account the sum of probable and confirmed cases outbreak indicator. The chikungunya cases included in this analysis come from the Individual Epidemiological Notification, so they are not the total of cases that occurred during the outbreak (16). For Zika, individual notification was performed on many more patients therefore a higher proportion of cases were included in the analysis.

\section{Conclusions}

In conclusion, despite certain drawbacks in the registration of cases in the surveillance systems, the datasets were good enough to demonstrate the main epidemiological features of arboviruses outbreaks and encourage the application of the EWARS in order to mitigate their effects. The implementation of EWARS could reduce the magnitude of an outbreak and the resources invested in outbreak control.

\section{Declarations}

Ethics approval: Ethics Committee of the University of Freiburg $\left(\mathrm{N}^{\circ}-145 / 18\right)$

Consent for publication: The epidemiological data set were obtained from the Epidemiological Surveillance Systems of Norte de Santander (Colombia) and Mexico with authorization certificate.

Availability of data and materials: The data sets used and analyzed during the current study are available from the corresponding author upon reasonable request.

Competing interests: The authors declare that they have no competing interests.

Funding: The study was supported by the Special Programme for Research and Training in Tropical Diseases (TDR) and the Unit for Infectious Hazard Management (IHM) at the Department of High Threat Pathogens (PAT) at the World Health Organization in Geneva, Switzerland. Acknowledgements to the program "Scholarships for High Level Human Capital Formation for the Department of Norte de Santander - 2016" COLCIENCIAS number 753, for supporting RC. The article processing charge was funded by the German Research Foundation (DFG) and the University of Freiburg in the funding programme Open Access Publishing.

\section{Authors' contributions:}

RC. Design of the study, data collection and analysis, drafting manuscript

LH-A. Design of study, data analysis, drafting and correcting manuscript

DBV. Data collection and analysis, contribution to the text

GST. Data collection and analysis, contribution to the text

AK. Study design, data analysis, drafting and correcting the manuscript

\section{Acknowledgments}

The Departmental Institute of Health of Norte de Santander (IDS, Colombia) and the national vector-born-disease-control-programme (CENAPRECE, Mexico) were instrumental for transferring the surveillance datasets and the authorization for the analysis. We thank Institute of Hydrology, Meteorology and Environmental Studies-IDEAM, Colombia for providing the climatic data of the station in Cucuta.

\section{References}

1. World Health Organization. dengue guidelines for diagnosis, treatment, prevention and control treatment, prevention and control treatment, prevention and control. 2009. https://www.who.int/tdr/publications/documents/dengue-diagnosis.pdf. Accessed 3 Nov 2019

2. Gubler DJ. Dengue, urbanization and globalization: The unholy trinity of the 21 st century. Trop Med Health. 39. Supl.4. 3-11. 2011; doi: 10.2149/tmh.2011-S05.

3. Fredericks, A. C., Fernandez-Sesma, A. The burden of Dengue and Chikungunya worldwide: Implications for the Southern United States and California. Ann Glob Health. 80. 466-475. 2014; doi: 10.1016/j.aogh.2015.02.006

4. Kraemer, M. U., M. E. Sinka, K. A. Duda, A. Q. Mylne, F. M. Shearer, C. M. Barker, C. G. Moore, R. 780 G. Carvalho, G. E. Coelho, W. Van Bortel, G. Hendrickx, F. Schaffner, I. R. Elyazar, H. J. 781 Teng, O. J. Brady, J. P. Messina, D. M. Pigott, T. W. Scott, D. L. Smith, G. R. Wint, N. 782 Golding \& S. I. Hay. The global distribution of the arbovirus vectors Aedes aegypti and Ae. albopictus, eLife, 4, e08347. 2015; doi: 10.1371/journal.pone.0196811. 
5. World Health Organization. Estrategia de Gestión Integrada para la prevención y control del dengue en la Región de las Américas, 2017; http://iris.paho.org/xmlui/bitstream/handle/123456789/34859/OPSCHA17039_spa.pdf?sequence=8\&isAllowed=y. Accessed 15 Sept 2018.

6. National Observatory of Public Health. ONS-INS. Economic Costs associated with the care of patients with Chikungunya infection (CHKV) in Colombia. 2013; https://www.minsalud.gov.co/sites/rid/Lists/BibliotecaDigital/RIDE/IA/INS/Infografia-chk-ons.pdf Accessed 20 May 2018 (In Spanish)

7. Stahl, H. C., Butenschoen, V. M., Tran, H. T., Gozzer, E., Skewes, R., Mahendradhata, et al., Cost of dengue outbreaks: literature review and country case studies. BMC public health, 13, 1048. 2013; doi:10.1186/1471-2458-13-1048.

8. United Nations Development Programme UNPD. A Socio-economic Impact Assessment of the Zika Virus in Latin America and the Caribbean: with a focus on Brazil, Colombia and Suriname. 1-25. 2017; https://www.undp.org/content/undp/en/home/librarypage/hiv-aids/a-socio-economic-impactassessment-of-the-zika-virus-in-latin-am.html. Accessed 12 May 2018

9. Bowman L.R., Tejeda G.S., Coelho G.E., Sulaiman L.H., Gill B.S., McCall P.J., Kroeger A., et al. Alarm Variables for Dengue Outbreaks: A Multi-Centre Study in Asia and Latin America. Plos One. 2016; doi: 10.17605/OSF.IO/RTC5A.

10. Runge-Ranzinger S, Kroeger A, Olliaro P, McCall PJ, Sánchez Tejeda G, et al. Dengue Contingency Planning: From Research to Policy and Practice. PLOS Negl Trop Dis. 2016; doi.org/10.1371/journal.pntd.0004916

11. Bowman L.R., Donegan S., McCall P.J. Is dengue vector control deficient in effectiveness or evidence? Systematic review and meta-analysis. PLoS Negl Trop Dis., 10: e0004551. 2016; doi: 10.1371/journal.pntd.0004551

12. Organization WH. Operational guide: Early Warning and Response System (EWARS) for dengue outbreaks. 2018; https://apps.who.int/iris/bitstream/handle/10665/254668/9789241512053-eng.pdf. Accessed 15 Jan 2019

13. World Health Organization. Report. Global Strategy for dengue prevention and control, 2012-2020. 2012; http://apps.who.int/iris/bitstream/handle/10665/75303/9789241504034_eng.pdf;jsessionid=6EC7826FD89E643FE7EDC23C4B7C3B82?sequence=1. Accessed 8 June 2018

14. Hussain-Alkhateeb L., Kroeger A., Olliaro P., Rocklöv J., Sewe M.O., Tejeda G., et al. Early warning and response system (EWARS) for dengue outbreaks: Recent advancements towards widespread applications in critical settings. PLoS ONE 13(5): e0196811. 2018; doi: 10.1371/journal.pone.0196811.

15. National Administrative Department of Statistics - DANE - Colombia. Municipal Projections of Population 2005-2011 Sex and age groups. 2017; https://www.dane.gov.co/index.php/en/statistics-by-topic-1/population-and-demography/population-series-1985-2020.

16. National Health Institute, Colombia. External circular letter 1000-0001 (January 8-2015) Updating of guidelines for notification of cases of Chikungunya virus in Colombia, Phase II, 2015; http://manizalessalud.net/wp-content/uploads/2015/04/Circular-001-2015.pdf Accessed 5 Jun 2019 (In spanish)

17. Pan American Health Organization. Guide to comprehensive clinical care for patients with dengue. 2010. https://www.minsalud.gov.co/Documentos\%20y\%20Publicaciones/Gu\%C3\%ADa\%20para\%20la\%20atenci\%C3\%B3n\%20cl\%C3\%ADnica\%20integral\%20dt Accessed 28 Aug 2019 (In spanish)

18. Mattia M., Giorgio G., Piero P., Filipponi F., Solimini A., Caputo B., della Torre A., Rosà R., Merler S. Transmission dynamics of the ongoing chikungunya outbreak in Central Italy: from coastal areas to the metropolitan city of Rome, summer. Euro Surveill. 22. 17-00685. 2017; doi: 10.2807/15607917.ES.2017.22.44.17-00685.

\section{Tables}

\section{Dengue outbreak detection in Colombia and Mexico.}

\begin{tabular}{|c|c|c|c|c|c|c|c|}
\hline Country & Alarm Indicators & Sensitivity (\%) & PPV (\%) & No. of outbreaks & No. of Alarms & Alarm threshold & Lag week \\
\hline \multirow[t]{5}{*}{ Colombia } & Mean temp & 86 & 61 & 76 & 106 & 0.69 & 3 \\
\hline & Rainfall & 74 & 51 & 76 & 110 & 0.70 & 3 \\
\hline & Humidity & 80 & 60 & 76 & 102 & 0.65 & 3 \\
\hline & Probable cases & 91 & 50 & 77 & 141 & 0.75 & 5 \\
\hline & Multiple indicators* & 92 & 68 & 50 & 68 & 0.70 & 3 \\
\hline \multirow[t]{5}{*}{ Mexico** } & Mean temp & 81 & 72 & - & - & - & - \\
\hline & Rainfall & 87 & 65 & - & - & - & - \\
\hline & Humidity & 94 & 50 & - & - & - & - \\
\hline & Probable cases & 100 & 83 & - & - & - & - \\
\hline & Multiple indicators* & 84 & 77 & - & - & - & - \\
\hline
\end{tabular}

*Multiple indicators; temperature, precipitation \& humidity, PPV; Positive Predictive Value

** Values from Mexico taken from a previous period (see Hussain Alkhateeb et al. 2018)

Table 1. Sensitivity and PPV for Dengue outbreak detection using hospitalized dengue cases as outbreak indicator in relation to earlier changes in alarm indicators in Colombia and Mexico. 
Chikungunya outbreak detection in Colombia.

\begin{tabular}{|l|l|l|l|l|l|l|}
\hline Alarm Indicator & Sensitivity (\%) & PPV (\%) & No. of outbreaks & No. of Alarms & Alarm threshold & Lag week \\
\hline Mean temp & 77 & 71 & 13 & 14 & 0.80 & 10 \\
\hline Rainfall & 93 & 48 & 14 & 27 & 0.45 & 12 \\
\hline Humidity & 92 & 85 & 15 & 13 & 0.75 & 12 \\
\hline Multiple indicators* & 92 & 92 & 12 & 12 & 0.74 & 13 \\
\hline
\end{tabular}

*Multiple indicators; temperature, precipitation \& humidity, PPV; Positive Predictive Value

Table 2. Sensitivity and PPV for Chikungunya outbreak detection using probable chikungunya cases as outbreak indicator in relation to earlier changes in alarm indicators in Colombia.

Zika outbreak detection in Colombia and Mexico.

\begin{tabular}{|c|c|c|c|c|c|c|c|}
\hline Country & Alarm Indicator & Sensitivity (\%) & PPV (\%) & No. of outbreaks & No. of Alarms & Alarm threshold & Lag week \\
\hline \multirow[t]{4}{*}{ Colombia } & Mean temp & 100 & 11 & 2 & 19 & 0.05 & 10 \\
\hline & Rainfall & 50 & 54 & 2 & 7 & 0.05 & 6 \\
\hline & Humidity & 50 & 11 & 2 & 9 & 0.06 & 10 \\
\hline & Multiple indicators* & 100 & 14 & 2 & 14 & 0.05 & 10 \\
\hline \multirow{3}{*}{ Mexico } & Humidity (daylight) & 88 & 86 & 53 & 52 & 0.50 & 5 \\
\hline & Humidity (night) & 97 & 98 & 29 & 29 & 0.50 & 5 \\
\hline & Positive ovitrap & 92 & 97 & 25 & 25 & 0.40 & 5 \\
\hline
\end{tabular}

*Multiple indicators; temperature, precipitation \& humidity, PPV; Positive Predictive Value

Table 3. Sensitivity and PPV for zika outbreak detection using defined and probable cases as outbreak indicators in relation to earlier changes in alarm indicators in Colombia and Mexico, respectively. 